\title{
New analysis of the proper motions of the Magellanic Clouds using HST/WFPC2
}

\author{
Nitya Kallivayalil ${ }^{1}$, Roeland P. van der Marel ${ }^{2}$, Jay Anderson ${ }^{2}$, \\ Gurtina Besla ${ }^{3}$ and Charles Alcock ${ }^{3}$ \\ ${ }^{1}$ Pappalardo Fellow, MIT Kavli Inst. for Astrophysics \& Space Research, 70 Vassar Street, \\ Cambridge, MA, 02139, USA; email: nitya@mit.edu \\ ${ }^{2}$ Space Telescope Science Institute, 3700 San Martin Drive, Baltimore, MD 21218, USA \\ ${ }^{3}$ Harvard-Smithsonian Center for Astrophysics, 60 Garden Street, Cambridge, MA 02138, USA
}

\begin{abstract}
In HST Cycles 11 and 13 we obtained two epochs of ACS/HRC data for fields in the Magellanic Clouds centered on background quasars. We used these data to determine the proper motions of the LMC and SMC to better than $5 \%$ and $15 \%$ respectively. The results had a number of unexpected implications for the Milky Way-LMC-SMC system. The implied threedimensional velocities were larger than previously believed and close to the escape velocity in a standard $10^{12} \mathrm{M}_{\odot}$ Milky Way dark halo, implying that the Clouds may be on their first passage. Also, the relative velocity between the LMC and SMC was larger than expected, leaving open the possibility that the Clouds may not be bound to each other. To further verify and refine our results we requested an additional epoch of data in Cycle 16 which is being executed with WFPC2/PC due to the failure of ACS. We present the results of an ongoing analysis of these WFPC2 data which indicate good consistency with the two-epoch results.
\end{abstract}

Keywords. astrometry, galaxies: interactions, galaxies: kinematics and dynamics, Magellanic Clouds

\section{Introduction}

The Large and Small Magellanic Clouds (LMC \& SMC) at distances of $\sim 50 \mathrm{kpc}$ from the Sun, and $\sim 25 \mathrm{kpc}$ from the Galactic Plane, provide one of our best probes of the composition and properties of the Galactic dark halo, and have long been upheld as the poster-child for a strongly interacting system, both with each other and with the Milky Way (MW). It has commonly been assumed that the Clouds have made multiple pericentric passages about the MW, and indeed current formation theories for the Magellanic Stream, which may involve tidal or ram-pressure forces, require multiple pericentric passages in order to be viable stripping mechanisms (Gardiner \& Noguchi 1996, hereafter GN96; Connors et al. 2006; Mastropietro et al. 2005; Yoshizawa \& Noguchi 2003; Lin et al. 1995; Moore \& Davis 1994; Heller \& Rohlfs 1994; Lin \& Lynden-Bell 1982; Murai \& Fujimoto 1980).

However, recent high-precision proper motion measurements for the Clouds made by our group with two epochs of ACS High Resolution Camera (HRC) data in Cycles 11 and 13, where we measured the proper motion of LMC stars relative to background quasars, imply that the LMC tangential velocity is $\sim 370 \mathrm{~km} \mathrm{~s}^{-1}$, approximately $100 \mathrm{~km} \mathrm{~s}^{-1}$ higher than previously thought (Kallivayalil et al. 2006a, Kallivayalil et al. 2006b; hereafter K1 \& K2). The proper motion values of GN96, which have been adopted in all theoretical models of the formation of the Stream thus far, are not consistent with the new $H S T$ result. In particular, for the LMC there is a $7 \sigma$ difference. The values for the 
$\mathrm{SMC}$ are in more acceptable agreement ( $3 \sigma$ difference). The new measurements also indicate a significant relative velocity between the LMC \& SMC of $105 \pm 42 \mathrm{~km} \mathrm{~s}^{-1}$. This has been assumed to be closer to $\sim 60 \mathrm{~km} \mathrm{~s}^{-1}$ in theoretical models, i.e., approximately the value for the SMC to be on a circular orbit around the LMC.

These results have surprising physical implications which require a reconsideration of the formation mechanism for the Stream. These include the possibility that the LMC may only be on its first passage about the MW (Besla et al. 2007; hereafter B07). B07 demonstrated this by studying the past orbital paths of the LMC using our observed proper motions and errors in a $\Lambda$ CDM-motivated dark halo with a NFW profile (Navarro et al. 1996). This gave rise to starkly different trajectories for the LMC than those produced in a simple isothermal halo potential: even in the "best case" scenario (proper motion in the west direction, $\left.\mu_{W},+4 \sigma\right)$, the LMC only completes 1 orbit within $10 \mathrm{Gyr}$ and reaches an apogalacticon distance of $550 \mathrm{kpc}$ (see Figure 4 in B07). Subsequently, this has led to a series of papers exploring whether the LMC is indeed bound to the MW (e.g., Shattow \& Loeb 2009; Wu et al. 2008). Perhaps even more provocative is the possibility that that the LMC \& SMC may have only recently become a binary system (K2, although see Besla et al. these proceedings).

Such large motions were unexpected in light of our previous understanding of the MWLMC-SMC system, and it is therefore crucial that they be verified and further improved through the acquisition of additional data. Because of the large distances involved, even small differences in the proper motions can give vastly different orbits for the Clouds

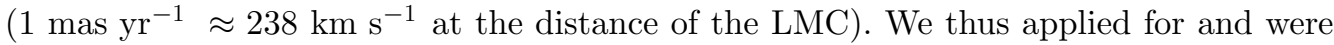
successful in getting a third epoch of snapshot imaging for our quasar-fields in Cycle 16. These executed with WFPC2 due to the failure of ACS. In this paper we present the preliminary results of an on-going analysis of these WFPC2 data. In $\S 2$ we present the WFPC2 data and analysis strategy with special attention to the relative size of the position errors vis-a-vis ACS. We describe the main sources of systematic error in both the ACS and WFPC2 data. In $\S 3$ we present results based on simple cuts aimed at minimizing these systematic errors, and discuss the expected overall improvement that this additional epoch affords. Since our analysis is still in the process of being refined, we present the results only in comparative fashion, both to our ACS-only two-epoch results (K1 \& K2), and to those of Piatek et al. 2008 (hereafter P08), who recently re-analyzed our ACS data using their own methods to obtain results that are consistent with ours. A brief summary and future prospects are presented in $\S 4$.

\section{WFPC2 data \& analysis}

\subsection{Description of observations}

In our first epoch program (Cycle 11, PI: Alcock), we imaged fields around 40 quasars behind the Magellanic Clouds (Geha et al. 2003) with the ACS High Resolution Channel (HRC) in snapshot mode (54 targets had been approved and we achieved a $74 \%$ completion rate). Each field was imaged in the V-band (F606W) with an eight-point dither pattern, to perform astrometry. We also obtained two I-band (F814W) exposures for each field, to allow the construction of color-magnitude diagrams (CMDs) and investigate any color-dependent effects. For the second epoch (Cycle 13, PI: Alcock) we proposed the same observational strategy (except without F814W). For the third epoch (Cycle 16, PI: Kallivayalil), however, due to the recent failure of ACS we proposed to use the WFPC2/PC. We requested the 40 targets observed in the first epoch in snapshot mode again. Our observational approach is similar to that in epochs $1 \& 2$. We used the V-band (F606W) filter on the Planetary Camera (PC) and a 5 or 6-point dither pattern. 

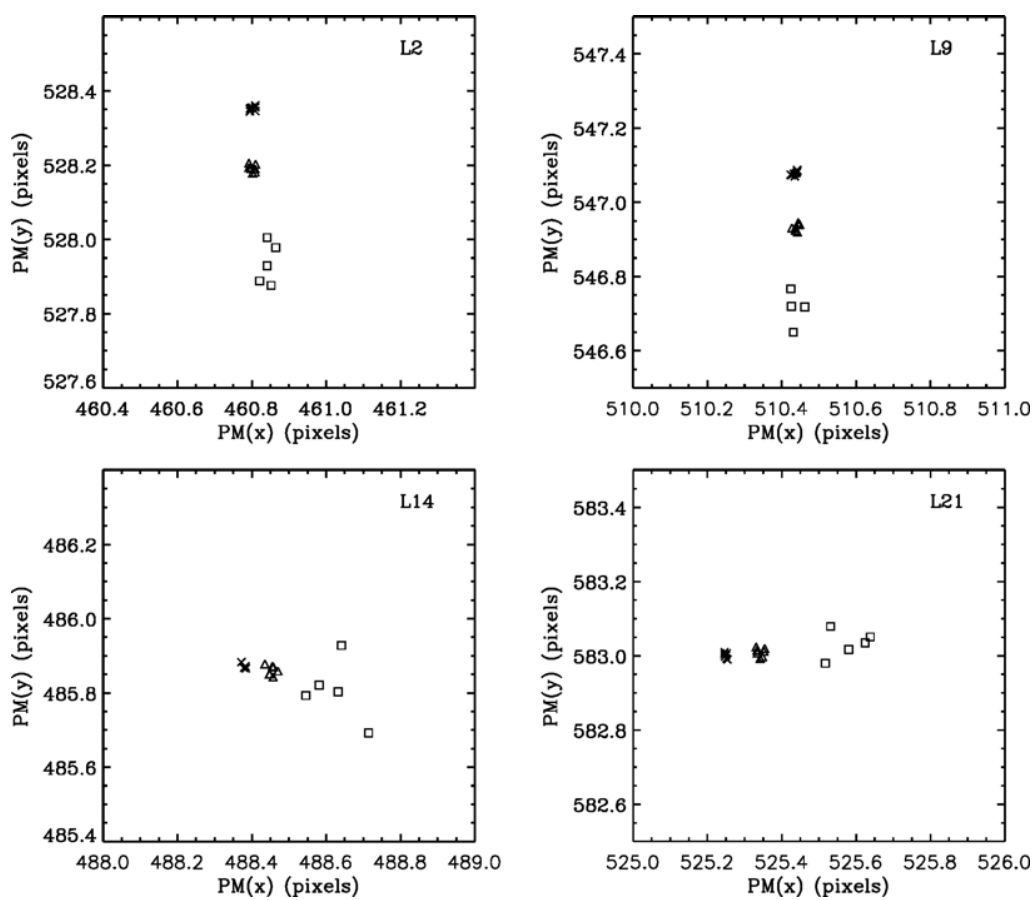

Figure 1. Positions, in a reference frame based on the 1st epoch images, of the quasar over time for 4 randomly chosen LMC fields showing ACS epoch 1 (crosses), ACS epoch 2 (triangles) and $\mathrm{PC}$ epoch 3 (open squares). The scatter per epoch gives the relative size of the rms errors in the position of the quasar relative to the star-field, given that the observation has been repeated many times per epoch.

The first concern in switching from the HRC to the PC was one of sensitivity. However our target quasars are relatively bright, ranging from $16.4 \leqslant V \leqslant 20$. The astrometric error is inversely proportional to the $S / N$ for the quasar. Aiming at $S / N \sim 100-200$ with the F606W filter yielded total science exposure times ranging from 2.8 to 20 minutes, thereby making these attractive snapshot targets. New data are still being obtained: so far we have obtained 16 out of an expected $\sim 20$ targets, 13 in the LMC and 3 in the SMC.

\subsection{Astrometric precision: Random errors}

There are many stars in each field, so the random errors in our transformations will be very small. The astrometric accuracy is therefore dominated by the accuracy with which we can measure the position of the quasar in each field relative to the surrounding stars. In Figure 1 we show the position of the quasar over time, in a reference frame based on the 1st epoch images, for 4 randomly chosen LMC fields (see K1 for definitions of these fields), showing ACS epoch 1 (crosses), ACS epoch 2 (triangles) and PC epoch 3 (open squares). The rms error in the position of the quasar is roughly 3 times as large for WFPC2 as for ACS ( $0.021 \mathrm{HRC}$ pixels versus $0.008 \mathrm{HRC}$ pixels).

\subsection{Astrometric precision: Systematic errors}

One of the main drivers in applying for a third epoch was to verify that there were no residual systematic effects in our measurements in addition to those that we already 

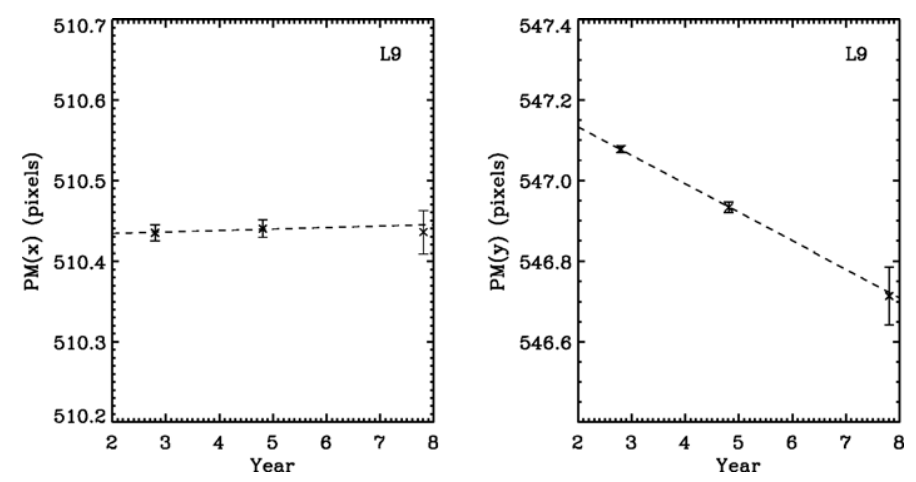

Figure 2. Geometrically-corrected positions ( $x$ on the left, $y$ on the right) for the QSO in field L9 for the three epochs of data. The line connects the first two epoch ACS measurements. The third epoch WFPC2 measurement is consistent with this.

accounted for in our error bars (e.g., residual geometric distortion solution errors). The third epoch does give us a handle on this as follows: there is always a straight line through two measurements, but if the third measurement doesn't line up then that is a clear indication of some systematic effect. Figure 2 shows geometrically-corrected coordinates for the quasar as a function of time, in the frame of the 1st-epoch, for field L9 for all three epochs. This is essentially a measure of the reflex proper motion of the field. The line connects the two ACS epochs and is not a fit. Thus even without detailed calculations it is easy to see that the third epoch is lining-up well, which rules out the presence of any major residual systematic errors.

We do know that there are astrometric errors due to charge-transfer-efficiency degradation (CTE) as well as other magnitude-dependent effects, mostly along the detector $y$-axis for ACS and along both $x$ and $y$ for the PC. These trends are small: $<0.02$ pixels for ACS, and $<0.03$ pixels for PC but are significant when considering the accuracy we are aiming for. Our strategy of observing our $N$ fields at random roll-angles of the telescope means that detector-frame-based errors average to zero as $N^{-1 / 2}$. However, they do introduce scatter. P08 showed that by accounting for these effects the rms scatter between fields can be reduced. Our current analysis also indicates that explicitly calibrating these effects can improve random errors.

\section{Results}

Since our analysis is still being fine-tuned, we focus in this section on demonstrating that there is generally good consistency between results from the third epoch of data and those from the first two epochs, once some simple cuts in magnitude-space to minimize systematic effects have been adopted (analogous to those in P08). Figure 3 is a field-byfield comparison for the LMC of the two-epoch proper motion results (blue squares) and the three-epoch results (pink diamonds). Proper motion in the north direction, $\mu_{N}$, is shown on top and proper motion in the east direction, $\mu_{E}$, is shown on the bottom. Lines show simple averages for the two-epoch case (dot-dashed), the three-epoch case (dashed), and for the P08 analysis of the first two epochs of these fields (solid). There is generally good agreement to within $1 \sigma$.

The proper motion values for the LMC quasar-fields with three epochs of data are shown in the $\left(\mu_{W}, \mu_{N}\right)$-plane in Figure 4. They are compared to the residual proper 

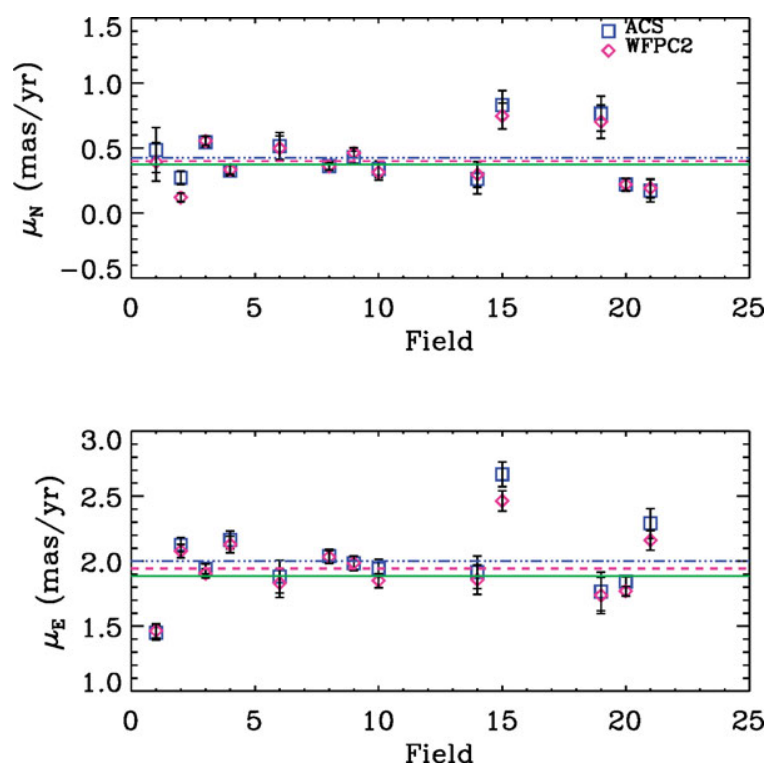

Figure 3. Field-by-field comparison for $\mu_{N}$ (top) and $\mu_{E}$ (bottom) for the LMC (see K1 for a definition of field numbers). Blue squares indicate two-epoch values, pink diamonds show three-epoch values. Lines are straight averages, dot-dashed for two-epoch, dashed for three-epoch and solid for the P08 analysis of these fields from our two-epoch data.
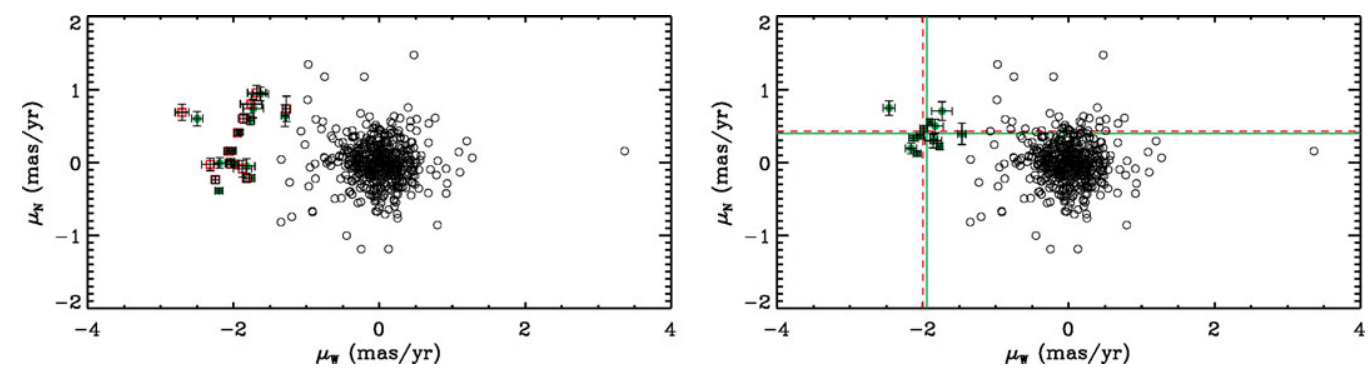

Figure 4. (Left) The observed PM $\left(\mu_{W}, \mu_{N}\right)$ for all the quasar fields with three epochs of data (filled circles). Over-plotted are the corresponding values from the two-epoch analysis (squares). (Right) The estimates $\mathrm{PM}_{\mathrm{est}}(\mathrm{CM})$ of the LMC center of mass proper motion after corrections for viewing perspective and internal rotation have been made for the three-epoch fields. The residual PMs of the LMC stars in all the fields are plotted with open circles in both panels. The reflex motions of the quasars clearly separate from the star motions. The straight lines mark the averages, dashed for the two-epoch analysis and solid for the three-epoch one.

motions of all the LMC stars that were used in the transformations. The stars are shown with open circles and the quasars with filled ones. Figure 4 (left) shows the observed $\mathrm{PM}$ values for all fields with three-epochs of data; filled circles show three-epoch analysis values while squares show the corresponding two-epoch analysis values. Figure 4 (right) shows the center of mass proper motion estimates, $\mathrm{PM}_{\mathrm{est}}(\mathrm{CM})$, just for the three-epoch fields, derived from the observed values after correcting for viewing perspective and internal rotation (see K1, van der Marel et al. 2002). The reflex motion of the quasars clearly separates from the star-fields in both panels. The solid line marks the average of the fields in the three-epoch analysis, while the dashed lines shows the corresponding average for the two-epoch data. For clarity, error bars are not plotted on the proper 
motions of the individual stars. However, these motions are generally consistent with zero given the error bars. The RMS errors calculated from the spread in Figure 4 (right) are currently a factor of $\sim 1.5$ better than our two-epoch results, but we expect to improve on this as we develop better models for CTE and other magnitude-dependent effects. SMC three-epoch results show a similar consistency with the two-epoch ones but are not shown here in the interest of brevity.

\section{Summary and future prospects}

We have presented an ongoing analysis of the proper motions of the Magellanic Clouds using a third epoch of WFPC2 data centered on background quasars. At present the rms error in the position of the quasar is roughly 3 times as large for WFPC2 as for ACS $(\sim 0.021$ pixels versus 0.008 pixels). However, with an improved method to deal with CTE and magnitude-related effects, and with the increase in time-baseline from 2 to 5 years, we expect final error bars for the proper motions that are smaller by a factor of $\ll 2$ from the two-epoch analysis: the two-epoch error bars in the north and east directions are

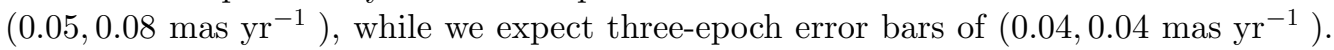
This will have several benefits, not the least of which is an important consistency check on our earlier results. This will allow us to better understand the orbit of the Clouds around each other. Combined with our understanding of the properties of the Magellanic Stream, this will allow us to better constrain the MW dark halo potential, as well as weigh into whether the LMC is indeed bound to the MW. Finally, we have a fourth epoch of observations scheduled in Cycle 17 with ACS and WFC3. The expected improvement in accuracy will continue to provide fundamental new insights into the unique and enigmatic Milky Way-LMC-SMC system.

\section{References}

Besla, G., Kallivayalil, N., Hernquist, L., Robertson, B., Cox, T. J., van der Marel, R. P., \& Alcock, C. 2007, ApJ, 668, 949 (B07)

Connors, T. W., Kawata, D., \& Gibson, B. K. 2006, MNRAS, 371, 108

Gardiner, L.T. \& Noguchi, M. 1996, MNRAS, 278, 191 (GN96)

Geha, M., Alcock, C., Allsman, R. A., et al. 2003, AJ, 125, 1

Heller, P. \& Rohlfs, K. 1994, A\&A, 291, 743

Kallivayalil, N., van der Marel, R. P., Alcock, C., Axelrod, T., Cook, K. H., Drake, A. J., \& Geha, M. 2006a, ApJ, 638, 772 (K1)

Kallivayalil, N., van der Marel, R. P., \& Alcock, C. 2006b, ApJ, 652, 1213 (K2)

Lin, D. N. C., \& Lynden-Bell, D. 1982, MNRAS, 198, 707

Lin, D. N .C., Jones, B. F., \& Klemola, A. R. 1995, ApJ, 439, 652

Mastropietro, C., Moore, B., Mayer, L., Debattista, V. P., Piffaretti, R., \& Stadel, J. 2005, MNRAS, 364, 607

Moore, B. \& Davis, M. 1994, MNRAS, 270, 209

Murai, T. \& Fujimoto, M. 1980, PASJ, 32, 581

Navarro, J. F., Frenk, C. S., \& White, S. D. M. 1996, ApJ, 462, 563

Piatek, S., Pryor, C., \& Olszewski, E. W. 2008, AJ, 135, 1024 (P08)

Shattow, G. \& Loeb, A. 2009, MNRAS 392, L21

van der Marel, R. P., Alves, D. R., Hardy, E., \& Suntzeff, N. B. 2002, AJ, 124, 2639

Wu, X., Famaey, B., Gentile, G., Perets, H., \& Zhao, H. 2008, MNRAS, 386, 2199

Yoshizawa, A. M. \& Noguchi, M. 2003, MNRAS, 339, 1135 